\title{
Background from radioactivity in dark matter detectors
}

\author{
Vito Tomasello ${ }^{* 1,2}$, Vitaly A. Kudryavtsev ${ }^{1}$, Matthew Robinson ${ }^{1}$ \\ ${ }^{1}$ Department of Physics and Astronomy, University of Sheffield, S3 7RH, UK \\ ${ }^{2}$ Physikalisches Institut, Eberhard Karls Universität Tübingen, Germany \\ E-mail: V.Tomasello@shef.ac.uk
}

\begin{abstract}
New generation dark matter experiments aim at exploring the $10^{-9}-10^{-10} \mathrm{pb}$ cross-section region for the WIMP-nucleon scalar interactions. Neutrons and gamma-rays produced in detector components are the main factors that can limit detector sensitivity. Radioactive background event rates from some detector components (such as copper and stainless steel), as well as from rock and concrete (lab walls), were estimated in this work for a hypothetical dark matter detector based on Ge crystals (for instance EURECA). Different shielding configurations (water, lead, polyethylene) were considered. Neutrons and photons were propagated to the detector using Geant4. Some requirements for the radiopurity of the materials were deduced from the results of these simulations. Thickness of shielding in different configurations and required gamma discrimination factor were investigated.
\end{abstract}

Identification of dark matter 2008

August 18-22, 2008

Stockholm, Sweden

\footnotetext{
* Speaker.
} 


\section{Introduction}

Background event rejection plays a crucial role in dark matter searches. Ordinary processes induced by cosmic-rays and radioactivity make the WIMP-detection very challenging, since its cross-section is predicted to be very low. Deep underground laboratories allow a reduction of cosmic-rays induced events by a factor of $10^{6}$ or more. However rock, concrete, shielding and any detector component contain some radioactive contamination. This results in a production of neutrons (from $\mathrm{U}$ and $\mathrm{Th}$ traces) and gammas (mainly from $\mathrm{U}, \mathrm{Th},{ }^{40} \mathrm{~K}$ ). They would be seen in the detector as nuclear and electron recoils respectively. The former are more difficult to be distinguished from the WIMP-induced nuclear recoils, the latter can be discriminated using various techniques. Eventually neutron background can seriously compromise the detector sensitivity.

Experiments and theories suggest the $10^{-9}-10^{-10} \mathrm{pb}$ region is the probable one for the WIMPproton cross-section. Reaching such level of sensitivity requires tonne-scale mass for the detector. But at the same time all background sources from radioactivity need to be carefully monitored and suppressed. Background suppression and rejection strategies and requirements on the depth, amount of active/passive shielding, purity of materials, veto efficiency, etc., for a given experiment, can be developed by an extensive simulation work. High-Z materials and low-A materials are known to be efficient in suppressing gamma-ray and neutron backgrounds respectively. Other factors can influence the selection of appropriate materials, most important are radioactive contamination, availability and cost.

In this paper we aim to outline some results on the radioactive background rising from rock, shielding and some detector components. Different configurations and solutions were taken into account. The simulations were done for a dark matter experiment based on cryogenic detectors (Ge crystals), such EURECA [1]. The design of the experiment and underground laboratory to host it are not finalised yet. These simulations are contributing to the design of a new cryogenic dark matter experiment.

\section{Background simulations}

EURECA is expected to be placed in the new hall of Laboratoire Souterrain de Modane (L.S.M.). For these simulations the existing laboratory hall was reproduced (30 m length, $10 \mathrm{~m}$ width, $10 \mathrm{~m}$ height, the walls of the hall are covered by $30 \mathrm{~cm}$ thick layer of concrete).

Fig. 1(a) shows the detector consisting of two copper vessels (inner vessel with thickness of $0.5 \mathrm{~cm}$ and a mass of $139 \mathrm{~kg}$, and an outer vessel with a thickness of $0.5 \mathrm{~cm}$ and a mass of $181 \mathrm{~kg}$ ) containing $103.68 \mathrm{~kg}$ of Ge. Crystals are arranged in 27 floors, each containing 12 crystals with a mass of $320 \mathrm{~g}$ each. Crystals are similar to those described in Ref. [2]. Although EuRECA will contain also scintillation crystals, current studies are limited to Ge detectors only.

Unlike gammas, neutron production spectra (from $(\alpha, n)$ reactions) depend on the material and these spectra are difficult to measure. Neutron and gamma-ray yield and spectra from radioactivity, used here, are taken from Refs. [3, 4] respectively. Neutrons and gammas with appropriate energy spectra were transported and detected using GEANT4 [5]. 
(a)

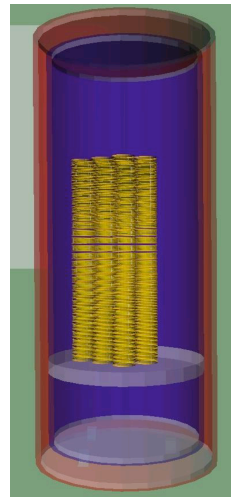

(b)
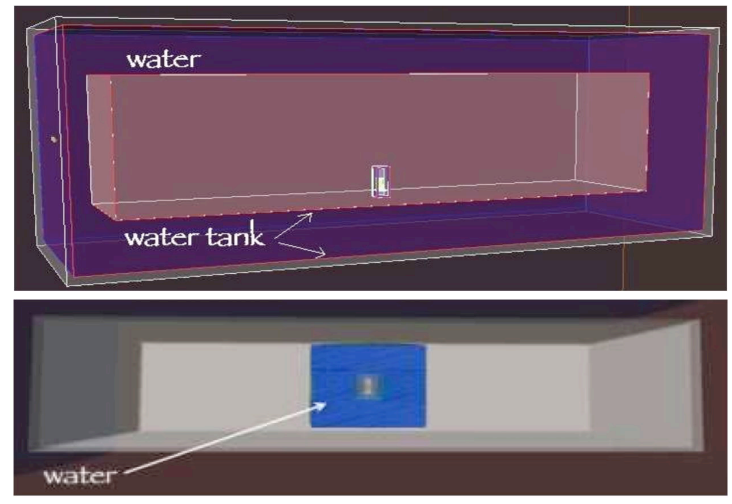

Figure 1: a) Schematic view of copper vessels and Ge crystals from GeANT4. b) View of the LSM laboratory from GEANT4 with water shielding the cryostat. Top: water is in a stainless steel tank along the laboratory walls; bottom: cryostat is immersed in water.

(a)
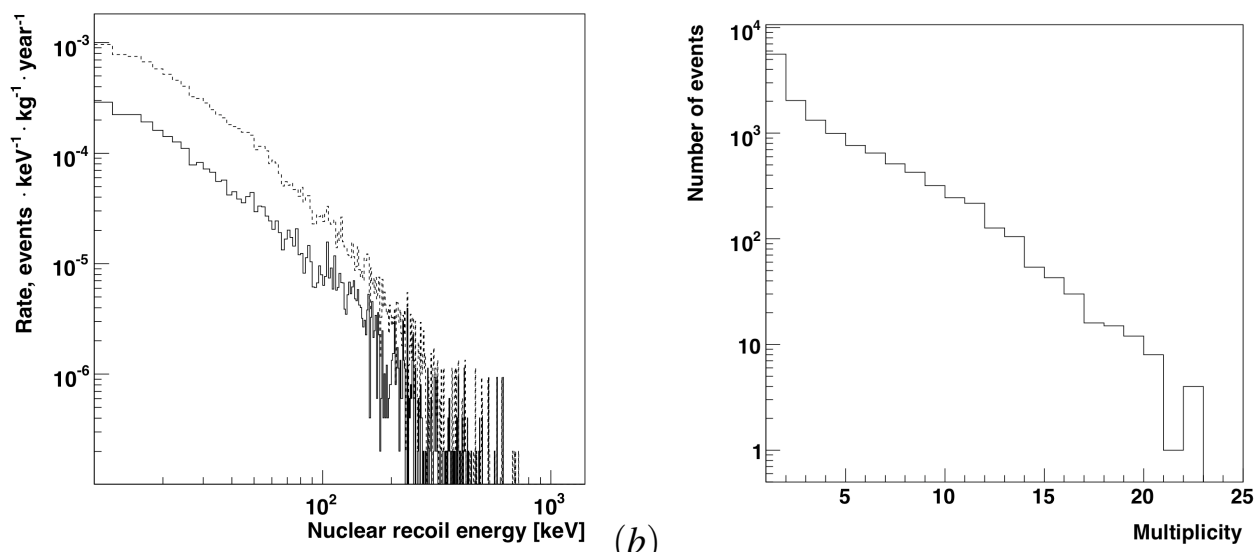

Figure 2: a) Energy spectrum of events including depositions for single nuclear recoils only, in $103.68 \mathrm{~kg}$ of Ge (solid curve). Dashed curve shows events with single nuclear recoils including the case when they are accompained by $e$-recoil depositions. b) Multiplicity distribution of energy depositions due to nuclear recoils. In both plots the polyethylene shielding surrounding the cryostat (mass $=21 \mathrm{t}$ ) is considered as radioactive source. An energy threshold of $10 \mathrm{keV}$ was assumed for each deposition.

\section{Results}

Table 1 summarises the expected rate of background induced events. Starting from the first column on the left the table reports the radioactive source considered along with its total mass and concentration of radioactive nuclei, then the shielding configurations examined, finally the electron and nuclear recoil event rates. Both rates account for single scatter events only, in the range 10-50 $\mathrm{keV}$ per year, assuming a threshold of $10 \mathrm{keV}$ for each energy deposition. Fig. 2 shows typical energy distribution of single nuclear recoils (a), and the multiplicity distribution of nuclear recoil events (b). WIMP signal is not featured by events with multiple recoils.

As background sources, we considered rock and concrete whose contamination level and com- 
Table 1: Background event rate per year at $10-50 \mathrm{keV}$ due to single nuclear recoils from neutron interactions in $103.68 \mathrm{~kg}$ of Ge.

\begin{tabular}{|c|c|c|c|}
\hline source & shielding & $\begin{array}{c}\mathrm{e}^{-} \text {-recoil } \\
\text { events }\end{array}$ & $\begin{array}{c}n \text {-recoil } \\
\text { events }\end{array}$ \\
\hline \multirow{3}{*}{$\begin{array}{l}\text { concrete } \\
1.9 \mathrm{ppm} \mathrm{U}, 1.4 \mathrm{ppm} \mathrm{Th}, \\
2.5 \times 10^{3} \mathrm{ppm} \mathrm{K}\end{array}$} & $20 \mathrm{~cm} \mathrm{~Pb}+50 \mathrm{~cm} \mathrm{CH}_{2}$ & - & 0.064 \\
\hline & $20 \mathrm{~cm} \mathrm{~Pb}+60 \mathrm{~cm} \mathrm{CH}_{2} \mathrm{O}$ & - & 0.125 \\
\hline & cryostat in water $2 \mathrm{~m} / 3 \mathrm{~m}$ & $1.03 \times 10^{5} / 1300$ & - \\
\hline \multicolumn{4}{|l|}{ stainless steel (lab walls) } \\
\hline $12 \mathrm{t}-1 \mathrm{ppb} \mathrm{U} / \mathrm{Th} / \mathrm{K}$ & none & $8 \times 10^{5}$ & 17.2 \\
\hline \multicolumn{4}{|l|}{ copper (cryostat) } \\
\hline $320 \mathrm{~kg}-0.01 \mathrm{ppb} \mathrm{U} / \mathrm{Th}, 10 \mathrm{ppb} \mathrm{K}$ & none & 6677 & 0.0308 \\
\hline \multirow{3}{*}{$\begin{array}{l}\text { stainless steel } \\
\text { (inner cryostat vessel) } \\
347 \mathrm{~kg} \text { - } 1 \mathrm{ppb} \mathrm{U} / \mathrm{Th} / \mathrm{K}\end{array}$} & none & $3.99 \times 10^{5}$ & 2.54 \\
\hline & $5 \mathrm{~cm} \mathrm{~Pb}+10 \mathrm{~cm} \mathrm{CH}_{2}$ & $1.54 \times 10^{4}$ & 0.042 \\
\hline & $10 \mathrm{~cm} \mathrm{CH}_{2}+5 \mathrm{~cm} \mathrm{Cu}$ & - & 0.130 \\
\hline \multicolumn{4}{|l|}{ polyethylene (inside the cryostat) } \\
\hline $366 \mathrm{~kg}-0.1 \mathrm{ppb} \mathrm{U} / \mathrm{Th} / \mathrm{K}$ & none & $1.12 \times 10^{5}$ & 0.288 \\
\hline \multicolumn{4}{|l|}{ polyethylene (inside the cryostat) } \\
\hline $462 \mathrm{~kg}-0.1 \mathrm{ppb} \mathrm{U} / \mathrm{Th} / \mathrm{K}$ & $5 \mathrm{~cm} \mathrm{Cu}$ & 819 & 0.333 \\
\hline
\end{tabular}

position are taken from [6]. The first row of Table 1 reports rates only for concrete since this contribute the most (97\%). Shielding configurations examined for this case were: i) lead and polyethylene (inside lead); ii) lead and water (inside lead); iii) water. Table 1 shows that $50 \mathrm{~cm}$ of $\mathrm{CH}_{2}$ or $60 \mathrm{~cm}$ of $\mathrm{H}_{2} \mathrm{O}$ can attenuate the $n$-recoil event rate to a negligeble level. Gamma-ray induced background was not simulated for this case, since it was already shown that $20 \mathrm{~cm} \mathrm{of} \mathrm{Pb}$ are able to suppress the event rate by 5-6 order of magnitude [7].

Works for extending the Modane Laboratory are scheduled for the next years and a new hall will host the EURECA detector. There is a possibility to use water to shield the cryostat from both neutrons and gammas. One solution can be a cryostat immersed in water, see Fig. 1(b). The thickness of the water shielding able to suppress the gamma-ray background was investigated. Beyond $3 \mathrm{~m}$ thick water shield the $e$-recoil event rate is attenuated significantly so that a discrimination factor of only about $2 \times 10^{3}$ is needed. Another opportunity could be having water around the lab walls, contained in a stainless steel tank (Fig. 1(a)). In this case the cryostat does not have any shielding from the radiation produced inside the hall. The benefit of this solution is an experimental hall free of any shielding and a direct access to the detectors. The thickness of water should be similar to that found in the case of water tank around cryostat. With 2 meter of water additional shielding inside the cryostat is required. The steel laboratory walls are, however, a potential source of background, since the detectors are not shielded from the radiation coming from this tank. The second row in Table 1 shows that this would require additional shielding or a discrimiation factor of $10^{6}$. Shielding, as well as any detector components, produces background radiation that, if not 
sufficiently controlled, would affect the detector sensitivity. We considered the cryostat for two different cases. In the first instance it is made out of copper only. As reported in Table 1 (third row) event rate is very low for $n$-recoils, while a discrimination factor of $10^{4}$ is needed for $e$-recoils. The case of an inner vessel made of stainless steel was also examined. Since the level of contamination of this material is higher, the cryostat in this simulation was enlarged in order to place extra shielding inside. In the last part of Table 1 are results for two different inner shielding configurations considered. The first configuration was $5 \mathrm{~cm}$ of lead and $10 \mathrm{~cm}$ of $\mathrm{CH}_{2}$ (after lead). In this case the rate of $e$-recoils would still be high and moreover events due to the radioactivity of polyethylene would dominate (next to last row of Table 1). The second configuration was $10 \mathrm{~cm}$ of $\mathrm{CH}_{2}$ and 5 $\mathrm{cm}$ of copper (after $\mathrm{CH}_{2}$ ). Last row of Table 1 shows that $5 \mathrm{~cm}$ of $\mathrm{Cu}$ suppress the rate of events from polyethylene by 2 orders of magnitude.

\section{Conclusions}

Simulations show that the background coming from the lab walls can be attenuated by $20 \mathrm{~cm}$ of $\mathrm{Pb}$ along with $50 / 60 \mathrm{~cm}$ of polyethylene/water. Alternatively 3 meter of water can be used. If concrete has a thickness around $30 \mathrm{~cm}$, then its event rate dominates over the background from rock. A lab hall surrounded by water needs a discriminations factor of $10^{6}$ for gammas to remove the background from the steel tank, but inner shielding can attenuate it. Nuclear recoil rate from a cryostat in copper is much less than 1 event/year and does not require additional shielding even for large-scale detector. Discrimination factor of $10^{4}$ is needed for $100 \mathrm{~kg}$ target. If the inner vessel of the cryostat is made of stainless steel, then background attenuation would require $5 \mathrm{~cm}$ of $\mathrm{Cu}$ inside $10 \mathrm{~cm}$ of $\mathrm{CH}_{2}$ with a discrimination factor of about $10^{4}$.

\section{Acknowledgements}

This work was supported by the ILIAS integrating activity (ContractNo. RII3-CT-2004506222) as part of the EU FP6 programme in Astroparticle Physics. One of us (V.T.) would like to thank ILIAS for the financial support of his PhD research.

\section{References}

[1] H. Kraus et al., Nucl. Phys. B (Proc. Suppl. ) 173 (2007) 168; see also these Proceedings.

[2] X. F. Navick, M. Chapellier, F. Deliot, S. Herve and L. Miramonti, Nucl. Instrum. and Meth. A 444 (2000) 361 .

[3] V. Tomasello, V. A. Kudryavtsev and M. Robinson, Nucl. Instrum. and Meth. A 595 (2008) 431.

[4] D. Budjas and L. Pandola. GERDA Report GSTR-07-010 (2007); L. Pandola, private communication.

[5] S. Agostinelli et al. (GEANT4 Collaboration), Nucl. Instrum. and Meth. A 506 (2003) 250.

[6] V. Chazal et al., Astropart. Phys. 9 (1998) 163.

[7] M. J. Carson et al., Nucl. Instrum. and Meth. A 548 (2005) 418. 